\title{
Les doublets synonymiques chez Hervé Burel (1905) ou comment casser l'entre-soi
}

Synonymic doublets in Hervé Burel's work (1905), or how to break down the barriers

\section{Nelly Blanchard}

\section{OpenEdition}

\section{Journals}

Édition électronique

URL : https://journals.openedition.org/lbl/1882

DOI : $10.4000 / \mathrm{lbl} .1882$

ISSN : 2727-9383

\section{Éditeur}

Université de Bretagne Occidentale - UBO

\section{Édition imprimée}

Date de publication : 1 février 2013

Pagination : 161-180

ISBN : 979-10-92331-00-4

ISSN : 1270-2412

\section{Référence électronique}

Nelly Blanchard, «Les doublets synonymiques chez Hervé Burel (1905) ou comment casser l'entresoi », La Bretagne Linguistique [En ligne], 17 | 2013, mis en ligne le 01 mai 2021, consulté le 22 mai 2021. URL : http://journals.openedition.org//bl/1882 ; DOI : https://doi.org/10.4000/lbl.1882

La Bretagne Linguistique est mise à disposition selon les termes de la Licence Creative Commons Attribution 4.0 International. 
Nelly BLANCHARD*

\section{Les doublets synonymiques chez Hervé Burel (1905) ou comment casser l'entre-soi}

\footnotetext{
L
} e style d'Hervé Burel dans son autobiographie Histor eur famill eus Breïz Isel ${ }^{1}$ est remarquable à plusieurs titres. Bien que l'auteur n'invente aucun procédé stylistique ou rhétorique, qu'aucun jeu novateur avec la langue bretonne ne caractérise son texte, il me semble possible de parler de style chez Hervé Burel dans la mesure où la lecture d'une page de son texte permet de reconnaître son auteur à coup sûr. Comment cela est-il possible? C'est en fait la présence, dans un même texte, d'un ensemble de procédés rhétoriques et discursifs particuliers qui en fait un texte original, produit d'un auteur non moins original : un mélange de tournures populaires, d'expressions imitées de la littérature orale, de style mondain, de vocabulaire archaïque et moderne, mais aussi d'emprunt de tons et de thèmes à la fois à la littérature populaire française du XIX ${ }^{\mathrm{e}}$ siècle - notamment aux romans-feuilletons - et au syndicalisme révolutionnaire, le tout sous la plume d'un paysan-poudrier devenu écrivain et associant dans un même texte souvenirs de famille et revendications sociales.

* Maître de conférences de celtique, CRBC (EA 4451), UBO/ueb

1. Hervé BuREL, Histor eur famill eus Breïs-izel / Histoire d'une famille de Basse-Bretagne, manuscrit d'Hervé Burel ; établi, traduit et présenté par Nelly Blanchard, Morlaix/Brest, Skol-Vreizh/CRBC, 2011, 616 p. 
Si l'on définit la rhétorique comme de l'inattendu discursif, Hervé Burel sait créer de la surprise et de l'étonnement. Certains auteurs le font en suscitant la suggestion, en soustrayant du sens pour amplifier celui que le lecteur lui-même devra y trouver, en réduisant la proximité des mots les uns avec les autres, créant ainsi une constante frustration fructueuse dans l'attente du lecteur, moyen à la fois de tenir son attention et d'éveiller son imagination. Le style de Burel, pour inattendu qu'il soit, est à l'opposé de ces pratiques : il est caractérisé par une rhétorique de l'ajout. L'auteur ajoute, glose, enchérit, en dit plus plutôt que moins. Finalement, Burel joue sur l'attente comblée et non frustrée du lecteur.

Dans cette rhétorique de l'ajout, c'est au phénomène de redondance lexicale, et tout particulièrement au procédé de doublet synonymique (également appelé couple ou binôme synonymique), que je propose de m'intéresser. Je souhaite analyser ce que sont ces doublets synonymiques et poser quelques hypothèses sur la raison et la manière dont cet antique procédé rhétorique latin a pu arriver sous la plume d'un paysan léonard bretonnant anticlérical à la toute fin du $\mathrm{XIX}^{\mathrm{e}}$ siècle.

\section{Une balance linguistique}

Je ne garantis pas l'exhaustivité du relevé que j'ai réalisé dans ce texte long de plus de 350 pages, mais je propose toutefois une description du phénomène à partir d'environ 200 expressions extraites du texte, des doublets synonymiques ou expressions apparentées mettant en pendant deux éléments rapprochés par l'auteur, deux éléments comme posés en regard sur les plateaux d'une balance.

\section{Les types de balances}

La synonymie absolue n'existant pas, cette redondance lexicale par doublet rapproche en fait des termes sémantiquement plus ou moins proches. Mesurer la distance qui les sépare ou les rapproche est une opération impossible. Ce n'est pas le niveau sémantique qui permet d'y voir des catégories, mais la motivation de ces rapprochements. Pour saisir certaines différences entre ces expressions, je distingue provisoirement le rapprochement de deux mots :

- de sens voisin : je nomme le procédé « doublet», 
- appartenant au même champ lexical, à la même notion : je le nomme «duo»,

- nécessitant une explication (définition, variation dialectale...) : je le nomme «doublet métalinguistique».

De plus, Burel rapproche parfois trois ou quatre termes : je les nomme «triplet/quadruplet» ou «trio/quatro».

Parmi les 201 expressions relevées, le procédé le plus couramment employé par Burel est le doublet synonymique : «trist ha glacharus», "diréol ha diréglet», "yac'h ha dispos», «poaniet hag afliget», etc. On en comptabilise 91 occurrences sur 201, soit plus de $45 \%$ du corpus étudié. 15 d'entre eux sont utilisés plusieurs fois (de deux à cinq reprises) comme le précise le tableau suivant :

\begin{tabular}{|l|c|}
\hline Mots mis dans la balance & Fréquence d'emploi \\
\hline Yac'h / dispos & 5 \\
\hline Joaius / kontant & 4 \\
\hline Sujed / reson & 4 \\
\hline Joaius / laouen & 3 \\
\hline Joa / kontantamant & 3 \\
\hline Joa / laouenedigezh (ou inverse) & 3 \\
\hline Gae / joaius & 2 \\
\hline Kontantamant / laouenedigezh & 2 \\
\hline Kontant / laouen (ou inverse) & 2 \\
\hline Trist / glac'harus & 2 \\
\hline Chagrin / poan-spered (ou inverse) & 2 \\
\hline Droug / poan (ou inverse) & 2 \\
\hline Puill / stang (ou inverse) & 2 \\
\hline Skuizh / fatig bras (ou liou ar fatig warnomp) & 2 \\
\hline Teñval / du (ou inverse) & 2 \\
\hline
\end{tabular}

On constate que ce tableau présente des groupes relativement homogènes et que, si l'on rapprochait «joa» et «joaius», «laouen» et «laouenedigezh» etc., on réduirait encore le nombre de mots fréquemment mis en balance. Si l'on considère par ailleurs que 52 formules de doublets sont des hapax, cela appuie l'idée que le procédé tel que l'emploie Burel est très productif et vivant, et pas seulement emprunté à un fonds figé. 
Les duos rapprochent des mots appartenant à un même champ lexical, mais ne présentant pas de véritable équivalence, mais plutôt un complément d'information sémantique : «beo ha yac'h», «keuz ha glac'har», "melancoli ha glac'har», "goelvan ha daeloù» etc. Dans ce cas, ce n'est pas tant la synonymie qui est activée, que la forme binaire ou balancée. Toutefois, si l'identité entre les deux éléments mis en relation n'est pas totale, mais partielle, le mécanisme, notamment rythmique, tend à les faire percevoir comme des doublets synonymiques. Ils sont au nombre de 54 dans le corpus étudié, soit $27 \%$.

Autre procédé très proche des doublets synonymiques : les doublets métalinguistiques qui font souvent appel à des explications sur un terme du jargon de la Marine, ou encore qui fournissent un renseignement sur la variation lexicale (dialectale ou diachronique). Ce procédé qui joue sur l'aspect synonymique et l'équivalence sémantique perd parfois la forme balancée du doublet synonymique. Ces doublets métalinguistiques comptabilisent 32 occurrences, soit $16 \%$ du corpus. Ils sont parfois équilibrés comme «ar maturiou, pé ar guerniou » ou complètement déséquilibrés comme :

an dross eus ar stur ; pé da lavaret éo, ar gorden péhini à zo stag ous ar stur, ac à deu enn dro da ziou rod, éguis ma lavarfen, enn dro da droéllen eur puns, ac à éném gorréspond pa droër ar rodou zé, évit lakat ar stur da dreï à gléïs pé a zéou, évit gouverni ar batimantchou, ac ho dérija évélsé étrézec an andret ma ves c'hoant. / la drosse du gouvernail, ce cordage attaché au gouvernail et qui entoure deux roues, comme pour la manivelle d'un puits, et qui transmet le mouvement lorsqu'on les tourne pour actionner le gouvernail à droite ou à gauche, pour gouverner les bâtiments et ainsi les diriger là où l'on veut.

À cette catégorie de doublets métalinguistiques, on pourrait ajouter les 9 exemples de traduction littérale dans lesquels l'auteur passe explicitement d'une langue à une autre comme «hard-labour - en anglais, da lavaret éo, labour forcet» ou "an douzérés, (la tondeuse)».

Enfin, si les balances à plus de deux plateaux existaient, on pourrait filer la métaphore en trouvant une place pour les triplets (6), 
trios (6), quadruplet (1), et quatro (1). Les triplets sont tous des échos des doublets les plus fréquemment employés :

- Joaius / laouen / gae (3)

- Skuizh / fatig / simpl (2)

- Poan-spered / chagrin / glac'har (1)

Les trios reprennent des termes déjà employés dans les doublets ou les duos:

- Bew / yac'h / dispos

- Chagrin / tristidigezh / melancoli / keuz / diegi

Les quadruplets et quatros sont en fait des sortes de doublets ou duos doubles :

- tud honest a léal, just à guirion.

- â yoa ous ho férsécuti, ous ho flastra, ous ho c'hignat - ous ho distruga

Géographie des procédés étudiés

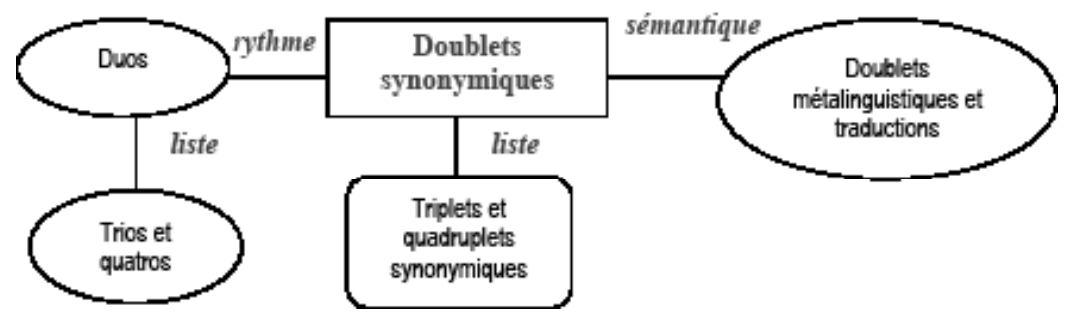

\section{Les plateaux de la balance}

Première caractéristique : sauf dans un cas («gant aoun da veza ê riskl $d a . . . /$ de peur d'être dans le risque de...»), les plateaux de ces balances accueillent des éléments de nature identique : deux noms, deux qualificatifs, deux groupes prépositionnels, deux propositions. Deuxième fait remarquable : la brièveté des éléments choisis semble faire partie de l'effet rythmique et stylistique recherché par ce procédé rhétorique. Il est à noter que les plateaux de ces balances ne permettent ainsi presque jamais la mise en regard de deux propositions entières. Seul ce duo métaphorique peut être considéré comme un pendant de deux propositions, bien que se combinent ici la proxi- 
mité sémantique et la métaphore : «or bués â zo continuéblamant en danger; neus német an neuden vreïn eus on d'iouall / Notre vie est continuellement en danger. Elle ne tient qu'à un fil pourri...»

Quelques verbes à l'infinitif entrent parfois en jeu, mais la quinzaine d'expressions concernées correspondent pour la plupart d'entre eux (13) à des doublets métalinguistiques, soit au jargon de la Marine lorsque l'auteur fournit des explications sur le sens de certaines manœuvres («apareilli, a zignifi, c'henc'h andret gant ar vatimant, évit mont en eul léac'h all / Appareiller signifie déplacer le bâtiment pour aller ailleurs»), soit sur des reformulations de variantes dialectales ( «ô torri, pé ô terri ; méïn / casser des cailloux»).

Finalement, les doublets synonymiques ou duos correspondent à la mise en regard de deux noms ou de deux qualificatifs (surtout de noms, parfois de verbes) : 96 cas de noms, soit presque $48 \%$ du corpus, et 81 cas de qualificatifs, soit plus de $40 \%$ du corpus. Même s'il arrive dans 7 cas qu'une préposition introduise les éléments («gant mes, à gant confusion / de honte et de confusion»), cela ne fait pas perdre l'impact de la concision des formules binaires.

\section{Le fléau de la balance}

Les expressions métalinguistiques et les traductions relient deux éléments par l'intermédiaire de moyens très variés :

- pe / ou («ar c'hap, pé an hent dré vor / le cap - ou chemin maritime-»),

- da lavared eo / c'est-à-dire («an den à vicher da lavaret éo ar médecin / l'homme de l'art, c'est-à-dire le médecin»),

- pe da lavared eo / ou c'est-à-dire,

- pe mar kirit / ou si vous voulez («mè a gomansas da ouèlapé mar kirit, da lenva / je me mis à pleurer»),

- a sinif / signifie («apareilli, a zignifi, c'henc'h andret gant ar vatimant, évit mont en eul léac'h all»),

- ar pezh a c'halver / ce que l'on nomme («ar glasfés er fons ; Ar pes a c'halver en gallec, l'algue verte. eun espés bézin glas / l'ulve dans le fond, une sorte d'algue qu'on appelle en français l'algue verte»),

- une virgule («dré ar porte-voix, dré an tuyo ma vés annoncet an névèzentiou eus ar machin var ar pont / par le porte-voix, 
le tuyau par lequel on communiquait entre la machine et le pont»),

- des parenthèses (« eur vorzéden kig moc’h, (jambon)»).

C'est toutefois le «pe» qui domine dans cette catégorie.

Par contre, dans les autres cas, les possibilités sont nettement réduites pour des raisons de formes et de rythme. La quasi-totalité des doublets synonymiques et des duos sont reliés par "ha / et» (ou parfois par son équivalent négatif «na») : 95\% des doublets synonymiques et $98 \%$ des duos. Parfois, les termes sont reliés par une simple virgule («ken rétt, ken ferm / si durs, si fermes») ou rien («mes allas siouas / mais hélas»).

Il semble donc que Burel distingue ce qui relève de la posture pédagogique et magistrale en utilisant un discours métalinguistique et explicatif, et la conjonction précisante et corrective «pe», de la posture d'éloquence par l'équilibre et la force de la formule binaire redondante et la conjonction additionnante «ha». Dans ce dégradé de procédés redondants, le cas des doublets métalinguistiques est intéressant car il se trouve à la jonction entre les traductions qui s'articulent souvent autour de «pe», mais proposent des éléments déséquilibrés, et les doublets synonymiques qui fonctionnent avec «ha» et équilibrent parfaitement les deux éléments : les doublets métalinguistiques s'inspirent du «pe» des traductions et de l'équilibre formel des doublets synonymiques. En fait, la forme balancée est conservée pour gagner en force et en effet rhétorique, mais le «pe» signale une distinction des éléments rapprochés. Les deux types de doublets ne rapprochent pas le même type d'éléments : les doublets métalinguistiques révèlent une conscience de l'auteur d'une dimension de variation géographique ou historique. Burel précise par un "pe», certes pédagogique, mais aussi sélectif, que tel mot se prononce de telle autre façon ailleurs, que tel mot vaut tel autre dans une autre région, et il signale encore que tel mot est en train d'être remplacé par tel autre. Par contre, dans le cas des doublets synonymiques, l'auteur additionne les mots, les associe de force. La perspective est donc différente. 


\section{Que pèse-t-on sur la balance des doublets?}

On y pèse des doublets et duos qui se rapportent pour la plupart à un nombre limité de champs lexicaux. Quatre champs lexicaux regroupent à eux-seuls $74 \%$ des occurrences. Il s'agit essentiellement pour l'auteur de donner du poids à l'expression de la souffrance vs bonheur, et du vice vs vertu. C'est donc essentiellement dans les parties narratives de son texte que Burel les emploie, et non dans les parties argumentatives.

\begin{tabular}{|l|l|}
\hline $\begin{array}{l}\text { Souffrance (souvent morale) } \\
\text { peine, chagrin, tracas, mal, tristesse, } \\
\text { détresse, amertume, angoisse, fatigue, } \\
\text { épuisement, affliction, contrition, honte, } \\
\text { confusion, tourment, difficulté, rudesse, } \\
\text { danger, misère, lourdeur, dégoût, regret, } \\
\text { mélancolie, plainte, soupirs, larmes. }\end{array}$ & $\begin{array}{l}\text { Bonheur } \\
\text { bonheur, jovialité, joie, satisfaction, soula- } \\
\text { gement, plaisir, étreintes, tendresse, cœur, } \\
\text { prospérité, pompes. }\end{array}$ \\
\hline $\begin{array}{l}\text { Vice } \\
\text { désordre, dévergondage, orgueil, fierté, } \\
\text { toupet, hardiesse, arrogance, dédain, } \\
\text { méchanceté, haine, félonie, cruauté, per- } \\
\text { fidie, fourberie, rigueur, rudesse, mépris, } \\
\text { froideur. }\end{array}$ & $\begin{array}{l}\text { Vertu } \\
\text { bonne conduite, capable, attention, précau- } \\
\text { tion, courage, vaillance, bravoure, loyauté, } \\
\text { franchise, droiture, justice, ordre, sagesse, } \\
\text { honnêteté, fermeté, honneur, bonne vo- } \\
\text { lonté, bonté. }\end{array}$ \\
\hline
\end{tabular}

Les autres expressions se rapportent à la santé ${ }^{2}$, au temps qu'il fait $^{3}$, à $1^{\prime}$ 'esprit ${ }^{4}$, à la description physique de personnages ${ }^{5}$, enfin restent "gis ha kustum / habitude et coutume», «inutil ha vean / inutile et vain» et «voyag hir ha koustus / voyage long et coûteux».

Ces balances permettent normalement de peser des synonymes. La synonymie absolue n'existant pas, il s'agit de s'interroger sur l'écart qui sépare les mots rassemblés. Ce ne sont pas les dimensions

2. Yac'h ha dispos, skiant vad ha réson, ententamant pe skiant an den, klenved lous ha danjerus, beo ha yac'h, lard ha yac'h, fresk ha diskouizh, , kreskiñ a gorf hag a oad, yec'hed ha prosperité.

3. Du ha tenval, kaer ha magnific, amzer yen ha rust, sioul ha brao.

4. Hervez ho c'hoant ha faltasi, ep sujed na réson, gant sujed ha gant reson, sclaeroc'h ha patantoc'h, goulenn excus ha pardon, bezit sur ha certen.

5. Ken rett (ha) ken ferm, kaer ha charmant kenañ, voues distinct ha skiltrus, coant ha capabl, ker flour ha ker mignon, bian ha dister, moues spontus ha gourdrousus. 
dialectale, diachronique ou de pratique de jargon qui motivent cet emploi puisque Burel se tourne alors vers d'autres dispositifs. Il semble que ce qui sépare les deux mots de la balance relève souvent de la variation linguistique sociale et témoigne de niveaux de langue différents volontairement réunis. Il s'agit dans de très nombreux cas de réunir un terme penchant plutôt vers le pôle paritaire et un terme penchant plutôt vers le pôle disparitaire, autrement dit un terme de breton populaire et un terme du breton plus savant ou aristocratique, l'un représentant la caution du vécu et du vernaculaire, l'autre l'autorité. En voici quelques exemples : «dinec'h ha dirémors», «poaniet hag afliget», «kri ha dinatur», «sclearoch ha patantoch», «chans vad ha boneur», «kaër ha magnific», «joaius ha laouen», «allas siouas», «distinct ha skiltrus»... On note d'une part que cela met souvent en balance un terme breton et un terme français, et d'autre part que l'ordre d'exposition des termes ne semble pas avoir grande importance et que Burel emploie autant l'ordre disparitaireparitaire que l'ordre paritaire-disparitaire.

\section{La question des sources}

L'emploi de doublets synonymiques n'est pas une invention d'Hervé Burel, bien entendu. Ce procédé rhétorique existe depuis fort longtemps, mais il s'agit de comprendre quels sont les modèles qui ont influencé l'auteur dans cet emploi, par quels canaux cette pratique a pu se retrouver sous la plume de ce paysan-écrivain. Estce par le biais de l'oral ou de l'écrit? Est-ce par le biais du breton ou d'une autre langue? Est-ce un emprunt figé ou une pratique vivante?

\section{L'origine du procédé}

C'est à Rome et à la rhétorique classique, notamment à la prédication, que nous fait remonter cette question de l'origine du procédé. Ce sont les Pères de l'Église qui ont donné forme et force à cette manière d'exprimer ce que l'on a compris des saintes Écritures. Saint-Augustin a divisé son manuel de prédication qu'est De doctrina christiana en deux parties, donnant ainsi autant de poids au message qu'à la forme que doit revêtir le message en question : «L'interprétation de l'Écriture comprend deux choses : la manière de découvrir ce qu'on y doit comprendre, et la manière 
d'exprimer ce qu'on y a compris ». Il consacre ainsi le livre 4 de son manuel à la rhétorique en s'appuyant notamment sur la rhétorique de Cicéron. La distinction qu'il pose entre les trois styles de prédication que sont les genus grande, genus temperatus et genus humilis a marqué pour longtemps la tradition rhétorique puisque ce sont toujours sur ces styles sublime, tempéré et simple que les auteurs d'ouvrages d'art poétique et de rhétorique française, comme Domairon (1816), s'appuient jusqu'au XIX ${ }^{\mathrm{e}}$ siècle.

Pour autant, qui dit rhétorique ne dit par forcément ornementation, pour Saint-Augustin. Dans le chapitre X de son manuel, il expose ses idées au sujet de l'importance de la clarté dans le discours. Il affirme préférer une pensée nettement rendue à une pensée ornementée, et si un orateur «rejette les ornements, ce n'est pas pour devenir bas et rampant.» Il oppose ainsi une expression qui serait certes «latine et savante» mais mal comprise, à une «expression familière, qui sur les lèvres du vulgaire présente un sens clair et déterminé». Les doublets - comme aucun autre précepte rhétorique - ne sont mentionnés chez l'auteur, mais on peut imaginer qu'ils participeraient de cet effort de clarté linguistique, ainsi que, ce qui n'est pas dit ici, au rayonnement social du discours pouvant à la fois être perçu par les «savants» comme par les «vulgaires». Ce rayonnement social, les orateurs et écrivains latins l'exprimaient, entre autres, en montrant leur double culture, grecque et latine, car employer des doublets synonymiques permet non seulement de se faire comprendre du plus grand nombre, de rendre son discours attrayant, mais aussi de prouver par le langage son appartenance à l'élite en insérant régulièrement des mots d'origine grecque.

Le succès dans toute l'Europe de cette pratique des binômes synonymiques est lié à l'enseignement des Arts poétiques dont le support était la rhétorique antique ${ }^{6}$. Pour le domaine français, Claude Buridant a retracé «l'histoire de la grandeur et de la décadence de cette " figure" qui a joué un si grand rôle dans la pratique scripturaire

6. Certains chercheurs pensent que le procédé se serait d'abord répandu dans les textes juridiques et administratifs, avant de gagner la littérature ; d'autres pensent qu'il s'est répandu dans tous les genres en même temps. Voir Claude Buridant, «Les binômes synonymiques. Esquisse d'une histoire des couples de synonymes du moyen âge au XVII ${ }^{e}$ siècle», Bulletin du Centre d'Analyse du Discours, $\mathrm{n}^{\circ} 4$, 1980, p. 6. 
de toute la littérature européenne ${ }^{7} \gg$. Il décrit plusieurs motivations à l'emploi de ces doublets synonymiques :

- Il note que l'emploi de doublets, notamment pour les épithètes, fait parfois perdre de la valeur sémantique aux termes pour gagner en intensité, donnant une sorte de valeur superlative (on traduirait ainsi le doublet synonymique de la phrase «ils entrerent el palais qui mult ere riches et biax» [E. Faral, ch. 8, p. 2] par «magnifique ${ }^{8}$ ) ou une valeur de sincérité 9 .

- Un autre usage est celui qui permet l'introduction d'un mot peu usité ou savant que le deuxième membre du couple explique et dont il adoucit l'effet d'inaccoutumé. Buridant cite von Wartburg qui rappelle que Nicole Oresme introduit ainsi en français des mots comme «agent», «auditif» etc. en employant le procédé rhétorique dans les doublets «agent et faiseur», «la puissance auditive ou puissance de oïr» etc. ${ }^{10}$.

- L'emploi de formules devenues stéréotypées, de binômes figés, empruntés à un stock, efface l'effet explicatif et rend un sens global au-delà des traits de spécification des termes ${ }^{11}$. Cette sorte de réserve de formules nourrit, en français comme dans d'autres langues, principalement les champs lexicaux de la joie, la tristesse et la peur, d'une manière générale dans l'expression de l'émotion ${ }^{12}$. Leur emploi fréquent finit par tendre vers l'hypertrophie et le maniérisme.

- Enfin, l'aspect esthétique, soit rythmique, soit phonétique (allitérations, assonances), joue un rôle important chez certains auteurs, comme chez Montaigne ${ }^{13}$.

Les traductions du latin en ancien français révèlent un emploi très fréquent de doublets en langue-cible là où la langue-source n'avait qu'un terme, une coordination fréquente d'un terme qui

7. BURIDANT, op. cit., p. 5.

8. BURIDANT, op. cit., p. 12-13.

9. ID., p. 19.

10. Ramussen et von Wartburg, cités par BURIDANT, op. cit., p. 14-15.

11. BURIDANT, op. cit., p. 15-16. «Joie» exprimait plutôt un contentement intérieur et «leece» un transport extérieur, mais le doublet «Od grant joie e o grant leesce» dépasse ces spécificités.

12. ID., p. 18-19.

13. ID., p. 36-39. 
sert de noyau notionnel et d'un terme qui sert à la spécification (par exemple «perficere» est traduit par «feire et asscoier $\left.{ }^{14} »\right)$, un essai d'acclimatation de mots savants, d'un néologisme calqué sur le latin. C'est ainsi que le moyen français s'est enrichi de mots savants, par un autre moyen que la périphrase ou la glose. La nouveauté ne pouvant être radicale pour les auteurs et traducteurs médiévaux, ils ont innové en s'appuyant à la fois sur la caution du connu et sur l'autorité du latin ${ }^{15}$.

D'après Hildergard Tristram, le style tempéré, qu'on qualifie aussi de fleuri, comme celui de Saint-Hilaire de Poitiers (IVe siècle), aurait influencé les prédicateurs gaulois, mais également, en Angleterre et en Irlande, les prédicateurs en hiberno-latin puis en anglo-latin, puis se serait transmis dans les littératures déclamatives en langues irlandaise (vieil et moyen-irlandais) et anglaise (vieil et moyen-anglais). Il existe ainsi en vieil anglais des poèmes homilétiques qui contiennent, dans une large panoplie d'ornementations rhétoriques, un grand nombre de doublets. $H$. Tristram rappelle que ces textes étaient «oraux », c'est-à-dire composés à l'écrit mais pour servir à des prestations orales (lectures ou récitations), et destinés à des auditeurs et non des lecteurs. L'esthétique verbale et le contenu religieux comptaient autant l'un et l'autre, le culte étant perçu comme l'art de servir Dieu. Les doublets de variantes synonymiques faisaient donc partie de cet art ${ }^{16}$.

Il n'est pas étonnant que ce procédé relevant à la fois de l'éloquence, de la clarté et de la distinction sociale ait fait fortune dans un domaine qui relève aussi en grande partie de l'oralité et qui touche la population entière : le domaine juridique. Mais il semble qu'un autre ingrédient soit indispensable : une situation sociale diglossique.

14. ID., p. 21.

15. ID., p. 20-30.

16. Je remercie Hildegard Tristram pour toutes ces informations. Voir précisément Hildegard L.C. TRISTRAM, «Intertextual puns in Piers Plowman», Neuphilologische Mitteilungen, 84, 1983, p. 182-191 ; H.L.C. TRISTRAM, Early Insular Preaching, Verbal Artistry and Method of Composition, Österreicheische Akademie der Wissenschaften, Phil.-hist. Klasse, Sitzungsberichte, tome 623, Édition de la Commission celtique, ${ }^{\circ} 11$, Vienne, Édition de l'Académie autrichienne, 1995 ; H.L.C. Tristram, Sex aetates mundi. Die Weltzeitalter bei den Angelsachsen und den Iren. Untersuchungen und Texte, Heidelberg, Carl Winter, 1985, chap. 4. 
En effet, alors que le domaine français n'est pas concerné, le domaine juridique anglais l'est. C'est un domaine dans lequel la langue française a été la langue de la justice pendant des siècles et l'établissement des lois en langue anglaise aux XIVe et XV siècles, dans un contexte diglossique, ne s'est pas fait sans de nombreux doublets : law and justice, final and conclusive, general terms and conditions, free and clear, part and parcel, full and complete etc. Certains, comme Lord Westbury, Lord Chancellor britannique en 1864, ont estimé le procédé trop lourd et proposé que soient opérées des coupes drastiques dans ces textes de lois «too often redundant and pleonastic $»^{17}$ !

La Bretagne n'a pas échappé à cette vague esthétique de la prédication et de l'éloquence, entretenue et adaptée tout au long du Moyen Âge et pendant la Renaissance. Aussi les doublets synonymiques se retrouvent-ils dans les mystères bretons des XVe et XVI siècles, commandes de la nouvelle bourgeoisie urbaine bretonnante, mais aspirant à imiter la noblesse nourrie de culture latine et française ${ }^{18}$. Un relevé non exhaustif des doublets synonymiques dans la Passion (1530), le Mirouer de la Mort (1576) et Buez Santes Nonn (XVI ${ }^{\mathrm{e}}$ siècle) montre que leur distribution n'est pas égale, mais que tous ces textes en contiennent un grand nombre. C'est Buez Santes Nonn qui en propose le plus (plus d'une centaine), puis la Passion (environ 80) et le Mirouer (environ 70). Si certains doublets se retrouvent dans deux ou trois de ces textes, comme «rayson ha guyrionez», «poan ha bihanez», «anquen ha penet», d'autres sont plus originaux «cazr ha jolis», «carguet ha samet», "yudet ha garmet bras», «tiz ha buhan», "profit ha gonit», «louen ha dreu», «glan ha net», «net ha pur» etc. Certains doublets ou duos présents

17. Benjamin HEYDEN, «D'une culture de common law à une culture civiliste : la traduction des doublets juridiques anglais en français », dans Traduire la diversité (domaines littéraire, juridique, et des sciences de la vie), colloque international, Université de Liège, 6-8 mai 2010, article numérique : http://www.13.ulg.ac.be/ colloquetraduction2010/textes.html, au 3 septembre 2012.

18. Voir Yves Le Berre, La Passion et la Résurrection bretonnes de 1530, suivies de trois poèmes, Brest, CRBC, 2011, p. 52 et 54, et Yves LE BERRE, Entre le riche et le pauvre. La littérature du breton entre 1450 et 1650, Brest, Emgleo Breiz, 2012, p. 61. 
dans ces textes sont également employés par Burel : «trist ha hueru», «hirvoudi ha gouela» ${ }^{19}$.

\section{Jusqu'à Burel ?}

À la fin du XVII siècle, une nouvelle esthétique basée sur la mesure, la justesse et la précision prend le pas sur l'ancienne esthétique, et le mot juste et unique supplante le doublet synonymique devenu symbole de décoration inutile, de babélisme et de pensée soit vide, soit non maîtrisée ${ }^{20}$. Seuls le style curial des ordonnances et des édits administratifs, et la harangue auraient perpétué cette pratique ${ }^{21}$. Quels sont donc les canaux par lesquels ce procédé est passé de l'époque médiévale à une autobiographie populaire écrite en 1905 ?

En Bretagne, à la fin du XVII ${ }^{\mathrm{e}}$ siècle et au XVIII ${ }^{\mathrm{e}}$ siècle semble s'amorcer également un affaiblissement de l'emploi écrit du procédé qui reste connu, mais peu au goût des nouveaux écrivains et cadres de la Basse-Bretagne. Dans une parodie de sermon écrite par un futur avocat ayant fait ses classes de rhétorique au collège de Saint-Pol-deLéon puis à Brest, on en relève quelques exemples : Claude-Marie Le Laè, dans le Sarmon var varo a Vikeal Vorin (1766), emploie ainsi «da reflèchi a da sonjal», «er c'hréguic fur ha tempret mat», «continuomp a déomp ato», «pront ha prest», «fagot muchab a founnus», "prop a quempen», ce qui montre que les modèles rhétoriques latin et français continuaient à l'époque à être étudiés et travaillés, généralement en français et parfois en breton, comme c'est exceptionnellement le cas pour Le Laè, que son professeur plaignait de ne pas savoir suffisamment le français et entraînait donc à la versification en breton ${ }^{22}$. De la même manière, les Colloques français-breton de Quiquer en contiennent quelques exemples (notamment «yac'h ha dispos»), mais en assez petit nombre.

On pourrait penser que la littérature religieuse serait le principal vecteur du procédé, or ni la Vie des Saints, ni les sermons ${ }^{23}$ n'en

19. Je remercie Yves Le Berre pour m'avoir facilité cette recherche à partir de textes numérisés.

20. BURIDANT, op. cit., p. 52.

21. BURIDANT, op. cit., p. 51.

22. Je remercie Yves Le Berre pour ces informations.

23. Voir par exemple le corpus sur Loiz ar Menn (1900-1994), présenté par 
contiennent suffisamment pour avoir pu être un modèle pour Burel. Les cantiques n'en proposent pas beaucoup plus : sur environ 70 cantiques étudiés, seulement 9 doublets synonymiques ${ }^{24}$ comme dans cet extrait de Pegen kaer : "Pegen kaer ez eo Mamm Jezuz / Pegen dous ha trugarezus / Pegen mad ha madelezus / Qu'elle est belle la Mère de Jésus / Qu'elle est douce et miséricordieuse / Qu'elle est bonne et compatissante». La revue Feiz-ha-Breiz, bien qu'utilisant des procédés de redondance lexicale, penche du côté du métalinguistique - et donc pédagogique - plutôt que du côté des doublets : plutôt «ar verouri-scol; pe ar ferm-scol-ze / cette métairie-école ou cette ferme-école ${ }^{25}$ » ou «a bep prez, a bep armel / de chaque armoire ${ }^{26}$ » que de rares doublets comme «ed du puil hag aleiz / beaucoup de blé noir ${ }^{27}$ » ou «ker sclear ha ker splann / de manière si claire et si évidente ${ }^{28} \gg$.

Du point de vue de la langue quotidienne, Jules Gros a procédé au relevé d'un certain nombre de doublets, dans ce qu'il appelle le style populaire. Dans la partie sur le «renforcement de l'expression», il décline la répétition en plusieurs catégories, dont une sur les locutions binaires et tout particulièrement sur la «réunion de deux mots différents mais quasi-synonymes ${ }^{29} »$. Parmi les 38 exemples

Maryvonne BERTHOU, Prezegennoù beleien : ul lennegezh vrezhonek?, Mémoire de maîtrise, UBO, Brest, 2004.

24. Relevé : ra vo karet e peb amzer / Hag adoret war an aoter ; an trouz hag oll safar ar bed ; o ankenioù hag o enkrez; glac'har hag enkrez; er boan hag en anken ; an enorioù hag ar meuleudioù ; mil bennozh ha trugarez; fiañs ha feiz e-leizh.

25. $\mathrm{N}^{\circ} 33,16$ octobre 1865, p. 260, 261.

26. $\mathrm{N}^{\circ} 134,24$ août 1867, p. 237.

27. $\mathrm{N}^{\circ} 152,24$ avril 1865 , p. 164.

28. $\mathrm{N}^{\circ} 46,16$ décembre 1865 , p. 364.

29. Jules Gros, Le Style populaire, Brest, Emgleo Breiz, 1984, p. 74. En voici la liste : Nag ayaou nag ha-dall, ha bardi ha bardao, blén-ha-barr, na bugel nag hursel, an debr hag an dag, o tifuhal hag o tirohal, dishual ha digabestr, dous ha brao, nag an eur nag an amzer, na grig na grag, na gras na feson, nag hent na gwenodenn, hir ha paduz, o huchal hag o hopal, evel just ha rezon, na kar na par, etre keuz ha reuz, lamm-saill, o lammad hag o saillad, he lipenn war he lapenn, na mui na ken, pilet ha torbilet, a-buillou hag a-druillou, na blego na ne zoublo, plên-ha-brao, dre bres ha dre reuz, na sin na zeblant, o skrignal hag o c'hoarzin, na stumm na paramant, na sujet na rezon, sul-gouel, teo ha lard, na terrin na trohañ, na tomm na klouar, en e dortou hag en e gluchou, trankil ha brao, treud ha kastiz. 
proposés, on constate que 15 d'entre eux sont des doublets basés sur un jeu sur la sonorité (onomatopée ou homophonie) : ha bardi ha bardao, o tifuhal hag o tirohal, na grig na grag, he lipenn war he lapenn... Ceci apparaît également dans certains proverbes comme «Karoud heb bezañ karet a zo kriz ha kaled / Aimer sans être aimé est cruel et dur». Parmi les expressions restantes, certaines soit rapprochent des termes proches, mais pas synonymes comme «na tomm na klouar», soit sont davantage des doublets métalinguistiques comme «na mui na ken». Seuls sept doublets synonymiques du relevé de Jules Gros pourraient finalement être mis en parallèle avec la pratique des doublets chez Burel, comme «hir ha paduz», «evel just ha rezon», «na sujet na rezon», «na stumm na paramant», «na gras na feson». L'emploi d'expressions redondantes et de doublets existe donc dans la langue populaire, mais c'est davantage le rythme binaire et l'homophonie qui en ont fait des moyens d'appui linguistique, et moins les aspects sémantique et sociolinguistique, comme si un filtre formel avait opéré dans cet héritage.

Dans le domaine de la littérature orale, deux genres semblent se distinguer nettement quant à l'emploi du procédé. D'une part, dans la littérature chantée on trouve quelques exemples apparentés à ce procédé rhétorique, mais en petit nombre. La chanson sur feuille volante Metig, composée par Jean-Marie Le Scourre, en contient bien (par exemple : «an disparti 'oa kaled, c'hwero hag ankenius / la séparation fut dure, amère et poignante »), quelques rares gwerz ou chants à danser rappellent le «kriz ha dinatur» (par exemple Yannig Skolan pour les gwerz et Daou zen yaouank o vale comme danse fisel). D'autre part, le théâtre dit "populaire» des XVII et XVIII siècles, semble jouer le rôle de continuateur du procédé utilisé dans les mystères médiévaux. Si des traces écrites de certaines de ces pièces de théâtre «populaire» existent, elles ne doivent pas occulter le fait qu'il s'agissait là de simples supports écrits à des prestations orales mettant en scène des acteurs issus des populations locales où se jouaient les pièces, et destinées à la foule ${ }^{30}$. L'exploration non exhaustive d'une de ces pièces de théâtre, la Passion $d u$ Christ

30. Sur ce type de théâtre, voir Gwennolé LE MENN, Histoire du théâtre populaire breton, XVe-XIXe siècles, Rennes, Institut Culturel de Bretagne/ Skol/ Dastum, 1983. 
adaptée en 1724 par Yves Le Borgne de Pleudaniel, révèle un emploi très régulier du procédé ${ }^{31}$.

En m'appuyant sur l'importance que semble constituer le modèle littéraire oral dans la transmission de cet élément rhétorique, je proposerais une hypothèse, en rebondissant également à la fois sur la harangue mentionnée par Buridant comme étant l'un des genres ayant conservé ce procédé, et sur l'affirmation par l'auteur de l'Histor eur famill eus Brë̈z Isel de son ascendance et sa culture protestantes. Le protestantisme n'a pas été très développé en BasseBretagne : seuls le Sud-Finistère et la région de Morlaix ont constitué de véritables centres protestants. Toutefois, Guillaume Le Coat (1845-1914), de Trémel, a traduit la Bible en breton en $1890^{32}$. Sa mère s'était convertie au protestantisme vers 1859 sous l'influence des collaborateurs de John Jenkins et était devenue institutrice ambulante. Le Coat devint pasteur, défendait ses convictions religieuses et républicaines dans divers journaux, et publia entre autres des traductions du Nouveau Testament et de la Bible ${ }^{33}$.

La mise en pages de la traduction de la Bible par Le Coat mérite qu'on s'y attarde car entre les deux colonnes sur lequel le texte est disposé se trouve une colonnette dans laquelle l'auteur inscrit des

31. Je remercie Jean-Paul Le Rol de m'avoir transmis ce relevé fait à partir du manuscrit conservé à l'abbaye de Landévennec. Voici quelques exemples : meurbet nechet haquazi hanter foll ; nep asitans nasuport ; dam chuitat yué dam dilezel ; ho dlée hac ou cridit ; quazi ogos ; enpoan hac en pennet ; ar pannio antourmant hac an nech; na guelas tourmenty natretan croadur; poan hatourmant bras ; foetez (-t) ha flagellet; chadenet amaret gant querden ; nedléet caffet poan nan nep affliccion ; nepsort glachar nac anuy ; gant poan ha gant tourmant ; mapilat ha mascein ; en poan hac en morchet ; en doan hac en morchet ; bout crouguet taguet ous ar corden ; sicour asistans ; hep urs ha hep condu ; fé ha fidelitez; eur gar(re)c ferm eur men diaséet ; napenos napenguis ; gollo ha leternio ; remors ha huannat ; quent divat ha quent cry ; toret ha briset ; cruel ha cry; atourmanchou acri ha a estlam; entourmant hac en nech ; a libertinet fal hac andut impudic ; ho lubricitez ho diroll debordet ; an tulmut hag an trouz ; tiset attrapet ; larron / brigantet potred fal ; gant poan ha gant fatic...

32. Ar Bibl santel en pehini ema ar gonvenans koz hag an hini nevez (1890), rééd., Paris/Genève, 1897. Je remercie Ronan Calvez de m'avoir mise sur cette piste.

33. Voir la notice sur cet auteur dans Yves LE BERRE, La littérature de langue bretonne. Livres et brochures entre 1790 et 1918, Brest, Emgleo Breiz, 1994, vol. 2, p. 399-401. 
indications. Certaines de ces indications sont des références aux Évangiles, d'autres des variantes dialectales, d'autres des variantes synonymiques. Comme l'une des méthodes développées pour l'évangélisation des populations bas-bretonnes par les protestants de Trémel était basée sur des tournées organisées par des pasteurs ambulants qui, sans doute, haranguaient la population rassemblée ${ }^{34}$, on peut imaginer un orateur transformer ces gloses en doublets synonymiques. Par exemple, la première page de la traduction propose la phrase "Hag an douar a oa deuet dizoare / la terre devint informe" accompagnée de la glose «diform / informe» : il est tout à fait imaginable que, oralement, une telle phrase se transforme en une formule comme «Hag an douar a oa deuet dizoare ha diform». Ainsi aurait pu être transmise cette manière de rapprocher deux termes de sens proches et, ce qui est assez fréquent chez Le Coat, deux termes tentant la complémentarité entre un terme populaire et un terme savant ou bien un néologisme issu du courant de rénovation linguistique initié par Le Gonidec ${ }^{35}$, comme «asamblez / kevred», «rum dud / mabiez, geneliez», etc.

\section{Une balance de la justice?}

La question du contexte sociolinguistique diglossique ne semble pas être étrangère à la pratique des doublets synonymiques : érudits latins voulant se parer de grec; traducteurs médiévaux adaptant le français, l'allemand, l'anglais au latin savant; justice anglaise ne voulant ou ne parvenant pas à renoncer à la tradition française ; mystères médiévaux breton faisant miroiter la culture française et latine ; puis Burel, dans un contexte préparant la double acculturation de la masse de la population bas-bretonne, à la fois par l'instruction et par la diffusion du français, bascule qui sera plus nette après la première guerre mondiale; enfin mouvement qui se poursuit jusque dans le dernier tiers du $\mathrm{XX}^{\mathrm{e}}$ siècle dans les médias en langue bretonne, notamment par la voix de Fañch Broudic, qui tâche de s'adresser

34. Jean-Gabriel FichaU, Trémel, Centre du protestantisme en Bretagne au $19 e$ siècle, Mémoire de maîtrise, s.1., 1970.

35. Louis Dujardin, La vie et les œuvres de Jean-François-Marie-Maurice-Agathe Le Gonidec, grammairien et lexicographe breton, Brest, Imprimerie commerciale et administrative, 1949. 
à un public double, celui des bretonnants de naissance et celui des néo-bretonnants.

Mais Burel n'est selon moi pas un simple miroir de ce contexte diglossique qui se met lentement en place. Les motivations de l'auteur vont plus loin : Burel déjoue les attendus socio-discursifs de sa position. Il n'est pas investi d'une puissance sociale, alors il use de la rhétorique pour devenir maître des résolutions, des préoccupations et des sentiments de ses lecteurs. En effet, l'impact esthétique, attrayant et persuasif du procédé rhétorique relevant d'un style recherché, rythmiquement équilibré, sémantiquement appuyé, thématiquement emphatique donne aux formules de la solennité, de la légitimité et de l'autorité. Mais les doublets synonymiques procurent à Burel un autre pouvoir : ils jouent le rôle de «passeport» sociolinguistique, c'est un moyen de passer les frontières sociales en permettant l'adresse à différentes classes de la population. Ils appuient ainsi les motivations que l'auteur exprime clairement par ailleurs : dénoncer l'accaparement du savoir par les nobles et les gens d'Église, lutter contre l'injustice sociale et affirmer l'importance primordiale de l'instruction du peuple. Il n'est pas ici question de rompre avec le sens commun des mots dans un but littéraire et esthétique, mais d'utiliser la littérature pour rompre avec l'emploi commun des mots, changer les distances qui séparent les riches des pauvres, les savants des ignorants.

Dans le chapitre 11 du livre 4 de la Doctrine chrétienne, SaintAugustin écrit : «À quoi bon une clef d'or, si elle ne peut nous ouvrir? Et qu'importe une clef de bois, si elle nous ouvre, quand nous ne cherchons qu'à ouvrir ce qui était fermé ? Mais sous certain rapport, il en est de ceux qui s'instruisent comme de ceux qui mangent : pour prévenir le dégoût, il faut assaisonner les aliments même les plus nécessaires de la vie.» Burel, dans une optique révolutionnaire de défense du peuple, écrit dans un breton de registre élevé et souvent mondain, adopte une posture magistrale, et va même jusqu'à la confection de trousseaux de clés comportant, au choix, une clé d'or et une clé de bois, pour prévenir du «chacun chez soi / pephini en e blass ${ }^{36} »$. Burel réalise d'un seul mouvement un éloignement

36. Voir aussi le proverbe «Pephini en e renk, Laezh dous ha laezh trenk / Chacun dans son rang, Lait doux et lait aigre...». 
d'avec ses semblables et un rapprochement avec ses dissemblables, obligeant ces derniers à se rappeler que la rhétorique est plus qu'un savoir-dire ou un savoir-écrire, mais un aussi savoir-entendre : par sa voix, les gens du haut de la société peuvent et doivent entendre ce qu'il a à dire, ils doivent renoncer à la connivence qui entretenait et protégeait leur entre-soi. Burel expérimente ainsi une mise en scène des pouvoirs de la linguistique sur la sphère sociale par le biais, fort symbolique, d'une balance linguistique portant une revendication de justice sociale. 